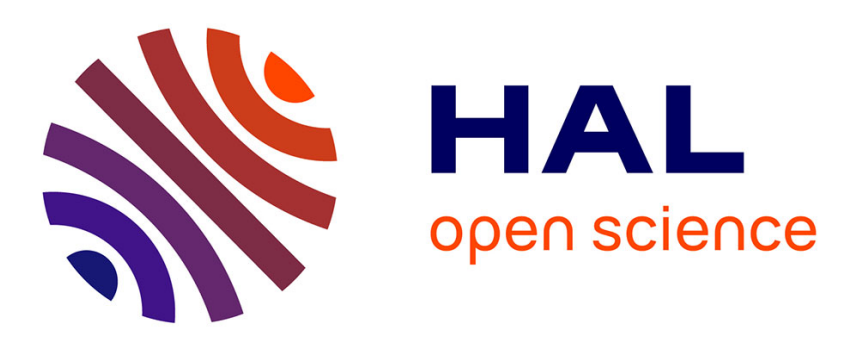

\title{
Compte rendu de " La Philosophie de la biologie avant la biologie. Une histoire du vitalisme" par Charles Wolfe
} Ghyslain Bolduc

\section{To cite this version:}

Ghyslain Bolduc. Compte rendu de " La Philosophie de la biologie avant la biologie. Une histoire du vitalisme " par Charles Wolfe. Bulletin canadien d'histoire de la médecine = Canadian bulletin of medical history, 2020, 10.3138/cbmh.391-092019 . hal-03161607

\section{HAL Id: hal-03161607 \\ https://hal.science/hal-03161607}

Submitted on 7 Mar 2021

HAL is a multi-disciplinary open access archive for the deposit and dissemination of scientific research documents, whether they are published or not. The documents may come from teaching and research institutions in France or abroad, or from public or private research centers.
L'archive ouverte pluridisciplinaire HAL, est destinée au dépôt et à la diffusion de documents scientifiques de niveau recherche, publiés ou non, émanant des établissements d'enseignement et de recherche français ou étrangers, des laboratoires publics ou privés. 
Bolduc, G. (2020). Compte rendu de « La Philosophie de la biologie avant la biologie. Une histoire du vitalisme » par Charles Wolfe, Bulletin canadien d'histoire de la médecine, 37(1), 308-311. DOI: 10.3138/cbmh.391-092019

\section{La Philosophie de la biologie avant la biologie. Une histoire du vitalisme}

Charles Wolfe

Paris : Garnier classiques, coll. Histoire et philosophie des sciences, 2019, 514 p, 56,00€

«Quel est le rapport entre l'interrogation philosophique du vivant et la constitution d'une science de la vie ?» (p. 197) C'est à ce problème que s'intéresse Charles Wolfe, qui ne prétend pas ici proposer des solutions définitives. Cette précaution est de mise vu l'ampleur de la tâche. L'ouvrage s'étend en effet de Descartes à la philosophie de la biologie contemporaine en passant par la controverse Leibniz-Stahl (partie 1), le « matérialisme vital » de Diderot (parties 1 et 2), le vitalisme de Montpellier (partie 3), Claude Bernard, Georges Canguilhem et la biophilosophie du $20^{\mathrm{e}}$ siècle (partie 4). L'analyse historico-philosophique prescrivait d'importantes exigences bibliographiques et méthodologiques dont l'auteur s'est généralement montré à la hauteur.

La problématique directrice de l'ouvrage est d'abord d'un intérêt épistémologique capital. L'auteur considère que la controverse soulevée au Siècle des Lumières par la question « qu'est-ce que la Vie ? » n'est pas étrangère à l'émergence de la biologie (vers le début du $\mathrm{XIX}^{\mathrm{e}}$ siècle). Il relève à cet égard les occurrences plus ou moins concomitantes des termes « organisme » comme « vocabulaire technique actif », « vitalisme » et « biologie » (p. 444). Manifeste dans les années 1740-1750, cette « crise ontologique » (p. 29) serait d'abord le fruit du débat entre Stahl et Leibniz, qui a généré plusieurs notions telles que la monade et les machines de la nature - ces machines qui seraient, contrairement aux machines artificielles, organisées jusque dans leurs moindres parties. Sur le plan métaphysique, on aurait ensuite assisté à une polarisation entre la vie en tant que substance - qui ne répondrait pas aux lois qui régissent la nature physique (l'âme stahlienne) - et la vie en tant que matière organisée. Mais l'auteur montre qu'une synthèse s'amorce rapidement au sein d'un courant matérialiste, 
Bolduc, G. (2020). Compte rendu de « La Philosophie de la biologie avant la biologie. Une histoire du vitalisme » par Charles Wolfe, Bulletin canadien d'histoire de la médecine, 37(1), 308-311. DOI: 10.3138/cbmh.391-092019

dont la provenance est en partie clandestine et qui tend à la fois : (1) à matérialiser l'âme humaine par une naturalisation de type cérébral (chez Fontenelle), volitionnel (chez Collins), etc., mais aussi par une médicalisation de la morale et de ses déterminations sociales (chez La Mettrie) ; (2) à spiritualiser la matière avec Maupertuis, qui postule l'existence de molécules vitales dotées de « désir, d'aversion, et de mémoire » (p. 178), ou alors à la vitaliser avec Diderot, qui attribue plutôt à ces molécules une «sensibilité sourde» (p. 187). Or pour qu'une controverse débouche sur une « production collective du savoir» (p. 21), l'auteur avance que la résolution de cette polarisation doit se faire sur la base d'un « cadre quasi-paradigmatique » (p. 35) pouvant regrouper divers phénomènes.

Engageons-nous sur les pistes tracées par l'auteur : si un tel cadre pouvait participer « à la définition des limites d'une nouvelle science » du vivant (p. 205), l'interrogation philosophique sur la spécificité de la «Vie » n'apparaîtrait-elle pas comme un moment fondateur de la genèse de la biologie? À la lumière de notre lecture, ce qui paraît mériter le statut de « cadre quasi-paradigmatique » d'une pensée biologique émergente est le mécanisme élargi, notion la plus féconde de l'ouvrage selon nous et qui devrait se trouver dans la boîte à outils de tout épistémologue de la biologie et de la médecine. Spectre de réduction des corps vivants et de leurs comportements fonctionnels à l'arrangement vital de leurs éléments matériels présumés, le mécanisme élargi se trouverait ainsi entre un mécanisme strict - qui n'admettrait que l'espace, la figure et le mouvement - et un « vitalisme substantiel » comme celui de Stahl. En vertu de la diversité des modèles qu'il supporte, ce cadre permet de rendre intelligibles des propriétés complexes de la chimie et de les articuler avec des explications mécaniques des phénomènes vitaux. Intégrant un newtonianisme analogique, il permet d'accepter dans un cadre théorique une propriété dont on observe les effets mais dont les causes sont provisoirement inconnues (comme la gravité), ce qui entraîne la suspension de l'analyse causale au profit d'une recherche de lois empiriquement éprouvées. C'est la 
Bolduc, G. (2020). Compte rendu de « La Philosophie de la biologie avant la biologie. Une histoire du vitalisme » par Charles Wolfe, Bulletin canadien d'histoire de la médecine, 37(1), 308-311. DOI: 10.3138/cbmh.391-092019

méthode qu'emprunte Haller dans son étude de l'irritabilité ; c'est aussi celle qui est à l'origine de la vis essentialis, principe responsable du développement selon C. F. Wolff.

Loin d'être antinomique, le mécanisme élargi engloberait donc un « vitalisme fonctionnel », selon lequel la «Vie » ne relèverait pas d'une substance immatérielle, mais de l'organisation particulière des parties d'un tout. Selon des vitalistes de Montpellier comme Bordeu et Ménuret, les propriétés générales de la vie émergeraient ainsi de l'interrelation de « petites vies » au sein de l'économie animale d'après le modèle de l'essaim d'abeilles. En somme, l'innovation conceptuelle de l'auteur et sa vision historique nuancée lui donnent les moyens de démythifier des stéréotypes généralement répandus dans certains milieux scientifiques et philosophiques. D'une part, on peut certainement être vitaliste sans « tricher » (p. 355) - c'est-à-dire sans postuler des forces vitales occultes. D'autre part, on peut être mécaniste sans abandonner « comme des jouets cassés » (p. 429) les systèmes qu’on aurait décomposés.

Dans la quatrième partie, l'auteur quitte le terrain épistémologique et tente d'évaluer la crédibilité de différentes « ontologies du vivant » qui ont été élaborées au cours des $\mathrm{XX}^{\mathrm{e}}$ et $\mathrm{XXI}^{\mathrm{e}}$ siècles. Il y affiche notamment sa préférence pour le vitalisme de Canguilhem, ce dernier ayant à la fois œuvré au sein d'une tradition biophilosophique prête à « dicter ses conditions à la biologie » en vertu de propriétés vitales « inaccessibles à la science quantitative » (p. 417) et refusé d'incorporer une métaphysique aux sciences biomédicales. Ce vitalisme naturaliste prendrait en compte les explications scientifiques tout en insistant sur l'irréductibilité de la vie comme expérience corporelle, existentielle et normative, de la vie créatrice de sens, de valeurs et d'un monde à habiter. Bien que ce projet nous semble globalement pertinent, cette dernière partie nous a paru parfois décalée par rapport au reste de l'ouvrage. Empruntant un style qui s'apparente à l'essai, l'auteur y exprime des jugements de 
Bolduc, G. (2020). Compte rendu de « La Philosophie de la biologie avant la biologie. Une histoire du vitalisme » par Charles Wolfe, Bulletin canadien d'histoire de la médecine, 37(1), 308-311.

DOI: 10.3138/cbmh.391-092019

goût et des commentaires parfois digressifs ou allusifs là où nous attendions des arguments dûment formulés.

En général cependant, cette histoire du vitalisme, principalement consacrée au contexte français, soulève des enjeux épistémologiques complexes et se donne les moyens rationnels d'en explorer de manière féconde les formes historiquement constituées. Passant des modèles cartésiens à leurs multiples « élargissements » matérialistes et vitalistes, elle est plus fondamentalement une histoire du mécanisme pré-biologique dont nous anticipons l'heureuse postérité.

Ghyslain Bolduc

Institut d'histoire et de philosophie des sciences et des techniques, Université Paris 1 